\title{
Integrando o ensino de astronomia e termodinâmica: explorando a zona habitável no diagrama de fases da água
}

\author{
Joint instruction of astronomy and thermodynamics: \\ exploring the habitable zone in the phase diagram of water \\ Maria Licia de Lima Farias, Marco Aurélio A. Barbosa*
}

Programa de Pós-Graduação em Ciência de Materiais, Faculdade UnB Planaltina, Universidade de Brasília, Planaltina, DF, Brasil

Recebido em 13 de Dezembro, 2016. Revisado em 19 de Março, 2017. Aceito em 29 de Março, 2017.

\begin{abstract}
Neste trabalho apresentamos uma abordagem em que tópicos atuais na área de astronomia podem ser utilizados para complementar o processo de ensino-aprendizagem em termodinâmica. Nesta direção, discutimos as condições astronômicas que potencialmente permitem o surgimento da vida tal qual conhecemos e introduzimos o conceito de Zona Habitável de maneira integrada ao ensino de termodinâmica, com enfoque nos diagramas de fases. Também apresentamos a lei de Stefan-Boltzmann e introduzimos o conceito de temperatura efetiva, por permitir uma formalização (ainda que de forma introdutória) dos limites da Zona de Habitabilidade em nosso sistema solar. Discutimos detalhadamente as condições de temperatura e pressão em cada um dos planetas rochosos em nosso sistema solar, comparando-os com o diagrama de fases da água líquida. Além disso, ilustramos como a pesquisa atual em astrobiologia poderá ajudar a encontrar planetas potencialmente habitáveis ao analisarmos dados da literatura com cenários possíveis para a atmosfera do candidato a exoplaneta Gliese 581g.

Palavras-chave: zona habitável, astrobiologia, diagrama de fases da água, habitabilidade
\end{abstract}

This work presents an approach where trendy astronominical topics can be used to complement the teachinglearning process in thermodynamics. In this direction, we present a discussion about the astronomical conditions that might potentially allow the emergence of life as we know, and the concept of Habitable Zone was introduced in pair with thermodynamical concepts, with emphasis on phase diagrams. Moreover, we introduced the StefanBoltzmann law and the effective temperature allowing a formal presentation of the Habitable Zone's limits in our solar system. The temperature and pressure conditions on each of the rocky planets in our solar system were discussed in detail and compared with the phase diagram of liquid water. Finally, atmospheric scenarios of the exoplanet candidate Gliese 581g were discussed to illustrate how the current research in astrobiology might help find potentially habitable planets.

Keywords: habitable zone, astrobiology, water phase diagrams, habitability

\section{Introdução}

Em uma carta a Heródotd ${ }^{1}$ o filósofo grego Epicurd ${ }^{2}$ defendia a ideia de um universo infinito, sendo incontável o número de mundos como o nosso [1. Este é o relato mais antigo que tomamos conhecimento sobre a existência de outros planetas, mas também é notório o caso de Giordano Bruno, que foi condenado à morte na fogueira por reivindicar a existência de uma pluralidade de mundos (além de ter cometido outras heresias contra a Igreja), durante a Idade média [2]. Outros filósofos e mais recentemente, cientistas, como Galileu Galilei e Jonas Kepler também se posicionaram a favor da existência de planetas fora do sistema solar. Entretanto, o primeiro

\footnotetext{
*Endereço de correspondência: aureliobarbosa@unb.br

${ }^{1}$ Historiador grego considerado o "pai da história"

${ }^{2}$ Filósofo grego
}

exoplaneta descoberto, 51 Pegasi b ou Dimidium ${ }^{3}$ (de acordo com a nomenclatura oficial da União Astronômica Internacional), só foi observado em 1995, orbitando uma estrela similar ao Sol na constelação de Pégasus, a 50 anos luz do nosso sistema solar 1]. Desde então, cerca de $3.472^{4}$ exoplanetas foram detectados orbitando, principalmente, estrelas dos tipos FGK 5 , sendo a maioria revelados através do método de trânsito, seguido por medições de velocidade radial ou efeitos de microlente gravitacional 3 .

\footnotetext{
${ }^{3}$ Nome vencedor de um concurso promovido pela União Astronômica Internacional, que no latim significa "metade" em uma referência a sua massa que é metade da massa de Júpiter

${ }^{4}$ Número confirmado de exoplanetas encontrados até 31/03/2017, data de revisão editorial deste trabalho.

${ }^{5}$ Estrelas são classificadas de acordo com sua temperatura e características espectrais. As classes de estrelas existentes formam a sequência OBAFGKM em ordem decrescente de temperatura.
} 
A detecção de um exoplaneta possivelmente habitável tem sido um dos principais propósitos de missões espaciais desenvolvidas pela NASA e a Agência Espacial Europeia (ESA), assim como um dos objetivos da astrobiologia moderna, por levantar a possibilidade de existência de vida fora do nosso planeta 4]. Neste século a astronomia tem avançado muito rapidamente com o uso de satélites e novos telescópios de grande envergadura e a busca por exoplanetas tem se beneficiado deste contexto. A sonda espacial Kepler, lançada em 2009, foi responsável por localizar até o momento 2.330 exoplanetas [5], enquanto futuros satélites como TESS (Satélite de Pesquisa de Exoplanetas em Trânsito), JWST (Telescópio Espacial James Webb) e PLATO (Oscilações e Trânsitos Planetários de Estrelas) vêm sendo projetados para a identificação e caracterização de planetas similares à Terra [4].

A astronomia, além de ser uma área da ciência que tem passado por grandes transformações, funciona como uma ponte entre o conhecimento científico e a sociedade, atraindo grande atenção do público [6]. Neste trabalho apresentamos uma proposta para o ensino de Astronomia e termodinâmica por meio de ferramentas interdisciplinares, ao entendermos que elementos diferentes atuando de forma integrada e harmônica, auxiliam na compreensão do conhecimento 7]. Com este intuito, a contribuição de fatores astronômicos e planetários para a existência de condições de temperatura e pressão que permitiram a existência de água líquida na Terra, será discutida tendo em vista a localização da Terra (bem como de outros planetas rochosos) no diagrama de fases da água.

Pretendemos com isso aperfeiçoar as concepções acerca de termodinâmica, abordando os conceitos de exoplaneta e Zona Habitável, além das condições planetárias para a origem da vida, de forma integrada ao estudo de conceitos ligados à termodinâmica, com ênfase na Lei de StefanBoltzmann e nos diagramas de fase.

Este artigo é organizado da seguinte forma, na seção 2 faremos uma síntese acerca do conceito de zona habitável, discutindo a Zona Habitável Galáctica, a Zona Habitável Circunstelar, a distinção entre habitabilidade instantânea e contínua, assim como as condições essenciais para a existência da vida. Na seção 3 discutimos a lei de StefanBoltzmann (que descreve a radiação emitida por um corpo negro) e a temperatura efetiva dos corpos celestes e na seção 4 apresentamos o diagrama de fases da água localizando neste diagrama condições atmosféricas típicas da superfície dos planetas rochosos do nosso sistema solar, bem como alguns cenários, vindos de modelos matemáticos da literatura, para o candidato a exoplaneta Gliese 581g. Nossas conclusões são apresentadas brevemente na seção 5

\section{Zona Habitável}

Uma das primeiras características analisada em relação aos exoplanetas é se eles estão localizados em uma região que possui condições de abrigar vida. Regiões deste tipo são denominadas de Zonas Habitáveis e são definidas como sendo um local em que (pelo menos) um ser vivo pode realizar suas atividades metabólicas.

Para serem considerados habitáveis na Via Láctea, os sistemas estelares devem permanecer a uma distância razoável do conturbado centro galáctico, uma região de intensa formação de estrelas, atividades de supernovas, emissão de raios gama, assim como perturbações gravitacionais 8]. Por outro lado, estrelas muito afastadas do centro estão em uma região de baixa metalicidade ${ }^{6}$, onde a abundância de elementos que formam os planetas terrestres é baixa. Portanto, estima-se que a Zona Habitável Galáctica, ilustrada pela figura 1 (a), ocorra em um raio de 7 à $9 \mathrm{kp} 7$ em torno do centro da nossa galáxia, onde se encontram estrelas formadas entre 4 e 8 bilhões de anos atrás [9].

Análogo à Zona Habitável Galáctica, a Zona Habitável Circunstelar é uma região ao redor de uma estrela com temperaturas favoráveis a presença de água líquida [4], visto que a água é o solvente necessário para a maioria

\footnotetext{
${ }^{6}$ Fração da massa de uma estrela que corresponde a elementos químicos que não sejam hidrogênio e hélio

${ }^{7} \mathrm{kpc}$ (quiloparsec): Unidade de comprimento utilizada para representar distâncias estelares. $1 \mathrm{kpc}$ é equivalente a $3,086 \times 10^{19}$ metros
}

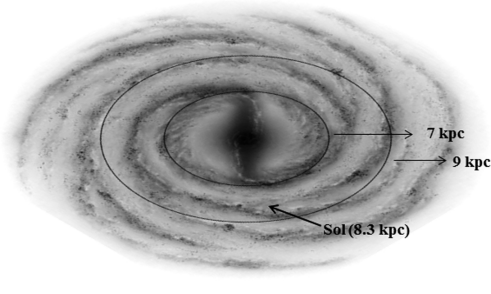

(a)

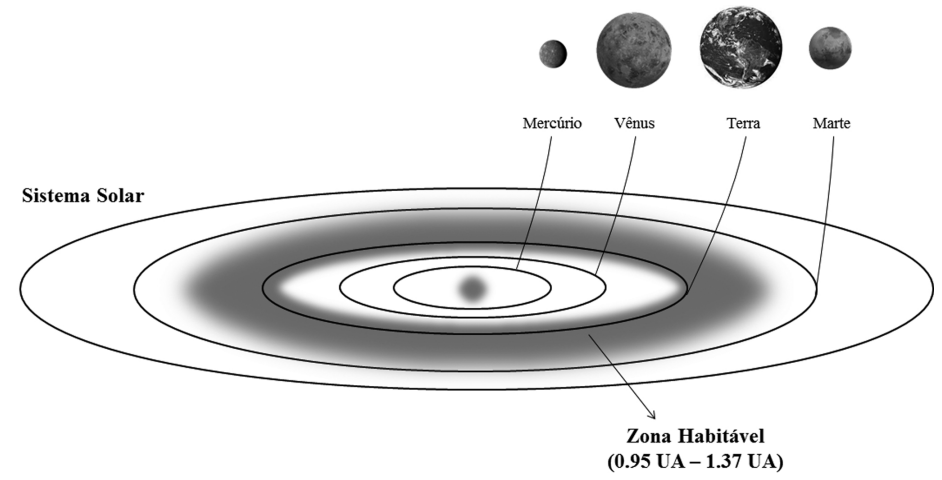

(b)

Figura 1: (a) Zona Habitável Galáctica e (b) Zona Habitável Circunstelar. 
das reações bioquímicas, resultando na manutenção do metabolismo dos seres vivos [9]. Convém mencionar que nos limitaremos à discussão das condições planetárias essenciais para o tipo de vida que conhecemos. Visões alternativas podem ser encontradas na referência [10].

Os limites da Zona Habitável Circunstelar, ou simplesmente Zona Habitável (ZH) variam com o tipo de estrela, sendo estas classificadas de acordo com sua temperatura e características espectrais. As classes definidas formam a sequência de letras OBAFGKM onde cada carácter representa um tipo espectral em ordem decrescente de temperatura. Estrelas do tipo O e B possuem os limites para a $\mathrm{ZH}$ mais longe da estrela quando comparadas ao nosso Sol, que é tipo $G$, enquanto as mais frias, dos tipos $\mathrm{K}$ e M, apresentam as fronteiras da $\mathrm{ZH}$ mais próximas à estrela. No Sistema Solar, a zona habitável, representada pela figura 1 (b), é determinada pela evaporação da água na borda inferior e pela formação de nuvens de $\mathrm{CO}_{2}$ na borda exterior, sendo estimada em uma distâncid ${ }^{8}$ de 0,95 UA à 1, 37 UA do Sol [11].

Para uma melhor clareza na compreensão do conceito de zona habitável, definiremos os conceitos de habitabilidade instantânea e habitabilidade contínua. A habitabilidade instantânea ocorre quando existem condições planetárias para as atividades vitais de pelo menos um organismo em um intervalo de tempo. Para que isto ocorra, deve-se satisfazer (pelo menos) as seguintes condições [9]:

- presença de água líquida;

- disponibilidade de energia para a manutenção, crescimento e reprodução dos organismos;

- existência de seis elementos da Tabela Periódica: carbono, hidrogênio, oxigênio, nitrogênio, fósforo e enxofre (CHONPS) 9

Já a habitabilidade contínua ocorre quando há a preservação das condições de habitabilidade instantânea, permitindo a existência de água líquida em um corpo celeste durante um período geológico de tempo, ou seja, milhões de anos.

A distribuição de água líquida é utilizada para classificar planetas e luas potencialmente habitáveis em dois tipos: aqueles que possuem água líquida na sua superfície e interior e os que abrigam água somente em seu interior. A Terra é um exemplo do primeiro tipo, em que a água líquida é mantida na superfície através de uma combinação entre o aquecimento interno do planeta, mas principalmente pela energia solar, devido a sua localização em relação ao Sol [9]. Quando a radiação estelar não é suficiente para a manutenção de água líquida na superfície, ela pode ser mantida por outras fontes de energia distintas da radiação luminosa. Por exemplo, as luas Europa e Ganimedes de Júpiter, assim como Encélado, em Saturno, podem ter oceanos sob extensas camadas de

${ }^{8} \mathrm{UA}$ (Unidade astronômica): Unidade de comprimento definida como a distância média da Terra ao Sol. O valor para 1 AU é de aproximadamente $1,5 \times 10^{11} \mathrm{~m}$

${ }^{9}$ Estão presentes em todas as formas de vida aqui consideradas, formando as macromoléculas essenciais para a vida gelo em suas superfícies, mantidos na fase líquida devido ao efeito de marés que surge da interação entre os núcleos do planeta e de suas respectivas luas [9].

Atualmente, parte significativa do trabalho dos astrobiólogos consiste em definir quais são os fatores que permitem a existência de água líquida e fotossíntese em um planeta, visto que estes potencialmente podem levar a emergência de vida multicelular. Podemos separar estes fatores em planetários e astronômicos, como listado abaixo e representado pela figura 2 .

Principais fatores planetários:

1. massa/densidade: influencia na composição atmosférica, determinando a perda ou a retenção de materiais voláteis;

2. atmosfera: o balanço de energia de um planeta é fortemente influenciado pela composição atmosférica e pelo albedo, que indica a reflexividade de uma superfície;

3. placas tectônicas: sustentam a temperatura média do planeta e permitem a reciclagem de substâncias como $\mathrm{CO}_{2}$;

4. campo magnético: atua como um escudo para a atmosfera do planeta protegendo-a contra a radiação solar e fluxos de plasma.

Fatores astronômicos:

5. tipo de estrela: determina a distância adequada para a existência de água líquida na superfície de um planeta;

6. rotação: determinante para a intensidade do campo magnético do planeta;

7. presença de satélite: interfere na regulação do clima do planeta;

8. impactos: podem afetar negativamente a habitabilidade provocando a vaporização de oceanos e extinções de espécies, porém também podem favorecer as condições para a vida aumentando a disponibilidade de nutrientes e energia.

No que se segue apresentamos a Lei de StephanBoltzmann para a radiação do corpo negro, que será utilizada para definir uma temperatura efetiva de um corpo celeste em torno de uma estrela e, posteriormente,

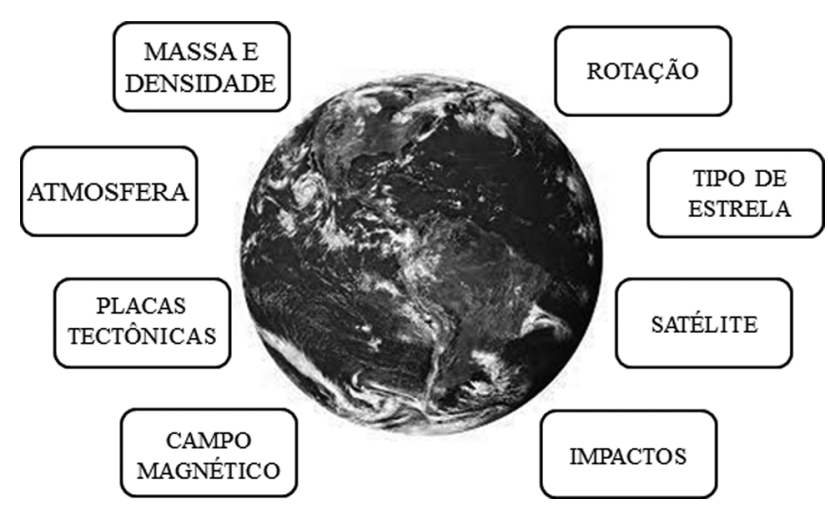

Figura 2: Habitabilidade Contínua. 
localizar aproximadamente a Zona Habitável no sistema solar 12]. Em seguida discutimos o conceito de transição de fases e localizamos os planetas rochosos do nosso sistema solar, assim como simulações da atmosfera do candidato a exoplaneta Gliese 581g no diagrama de fases da água. O objetivo é propiciar uma aprendizagem significativa utilizando instrumentos que ampliem a percepção dos alunos acerca da importância dos conceitos básicos da termodinâmica, através de uma proposta interdisciplinar integrando o ensino de astronomia e termodinâmica.

\section{Temperatura efetiva para corpos celestes em torno de uma estrela}

Como vimos acima, a presença de água líquida em uma escala de tempo geológica é o principal fator na determinação da habitabilidade contínua de um planeta. Uma diversidade muito grande de cenários pode ser construída considerando-se o conjunto de informações sobre os exoplanetas, no entanto, pode-se definir de forma simples as faixas inferiores e superiores da Zona Habitável em um sistema solar, utilizando-se a temperatura efetiva de um determinado corpo celeste. Ao considerarmos as trocas de energia entre o ambiente e um sistema, consideraremos uma estrela como um radiador de corpo negro perfeito, um corpo com emissão máxima de radiação eletromagnética devido às oscilações das partículas carregadas que compõem o objeto.

Neste cenário, pode-se estimar a potência total irradiada pela superfície da estrela através da lei de StefanBoltzmann:

$$
P_{S}=A_{S} \sigma T^{4}
$$

onde $A_{S}$ é a área da estrela, representada por $A_{S}=4 \pi R^{2}$ ( $R$ é o valor do raio estelar), $T$ é a temperatura média em sua superfície, e $\sigma=5,6697 \times 10^{-8} \mathrm{~W} / \mathrm{m}^{2} K^{4}$ é a constante de Stefan-Boltzmann. Para um corpo celeste localizado a uma distância $d$ de uma estrela a potência efetiva recebida é dada por:

$$
P_{e f}=A_{e f} \sigma T_{e f}^{4},
$$

onde $T_{\text {ef }}$ pode ser calculada de forma aproximada a partir de uma relação de igualdade entre a potência irradiada pela estrela e a recebida por uma esfera que contém a órbita (circular) deste corpo:

$$
4 \pi R^{2} \sigma T^{4}=4 \pi d^{2} \sigma T_{e f}^{4} .
$$

Desta forma, verifica-se que a temperatura efetiva cai com o inverso da raiz quadrada da distância $d$ à estrela:

$$
T_{e f}=\sqrt{\frac{R}{d}} T .
$$

Os limites de temperatura usualmente considerados como adequados para a existência da vida estão entre $-18^{\circ} \mathrm{C}$ a $121^{\circ} \mathrm{C}$, o que corresponderia, de acordo com a equação (4), a distâncias de $0,50 U A$ e 1,27UA [12].
Na figura 3 estão representadas as temperaturas efetivas encontradas através da equação (4) e os valores médios das temperaturas reais $T_{r}$ observadas na superfície dos planetas do sistema solar (no caso dos gigantes gasosos, a temperatura indicada corresponde a que é observada no limite superior da atmosfera). Notamos que, quando comparadas, as temperaturas dos planetas rochosos apresentam uma maior divergência entre as duas temperaturas, uma vez que estes possuem características particulares que afetam diretamente suas temperaturas, como será discutido na seção IV.

\section{Diagramas de fases}

Analisando livros didáticos de ciências do $6^{\circ}$ e $9^{\circ}$ ano do Ensino Fundamental [13], assim como livros do Ensino Médio,percebemos que as mudanças de fase são geralmente abordadas qualitativamente como ilustrado na figura 4 Neste gráfico, a adição ou remoção de calor e os estados iniciais e finais das substâncias são as principais informações consideradas.

Consideramos que ao utilizarmos o diagrama de fase da água para abordar tópicos em astronomia, possibilitamos

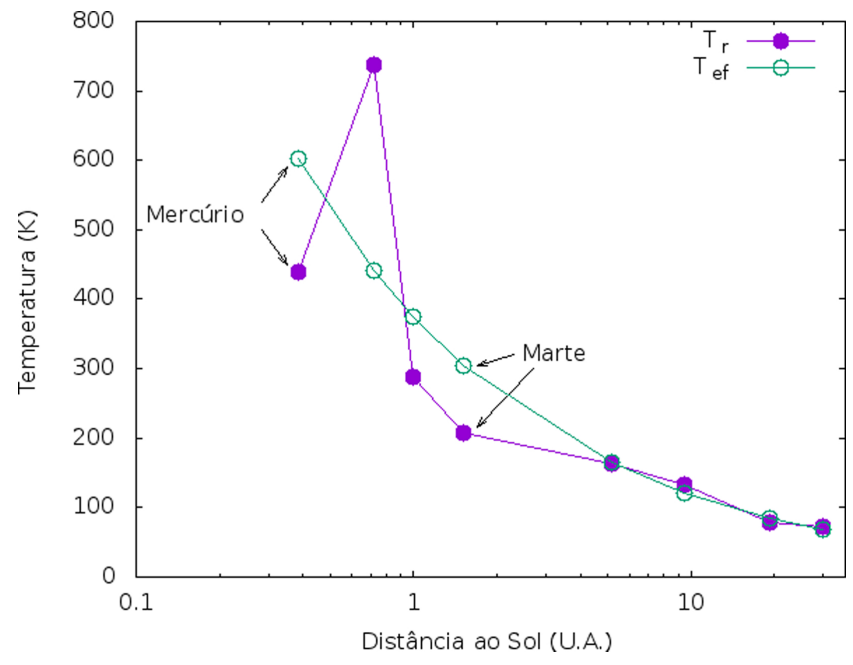

Figura 3: Temperatura efetiva dos planetas rochosos do sistema solar.

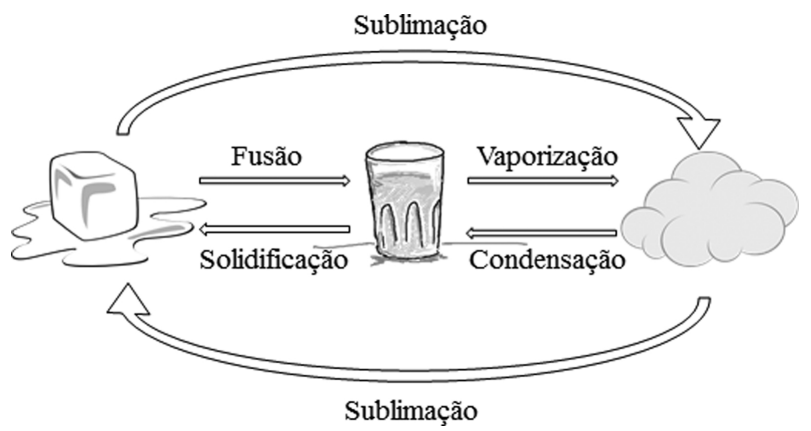

Figura 4: Esquema para o ensino de transições de fase, encontrado em livros didáticos do ensino fundamental. 
aos estudantes uma visão mais ampla sobre os processos de transição de fase, uma vez que o estudo do universo é um tema que mobiliza e desperta o interesse dos estudantes. Com este intuito, elaboramos um diagrama de fases no qual representamos os planetas rochosos do Sistema Solar e cenários possíveis para o candidato a exoplaneta Gliese 581 g [14, localizando-os de acordo com os valores médios para a temperatura e a pressão na superfície de cada astro (veja fig. 5). Os dados de pressão e temperatura média na superfície dos planetas rochosos foram extraídos do site da NASA [15] e os dados para o exoplaneta Gliese 581g foram extraídos de uma simulação computacional contemplando diversos cenários para todos os planetas próximos à zona habitável no sistema Gliese 581 [14.

Desta forma, permite-se aos estudantes a discussão sobre a habitabilidade de cada planeta, ou seja, sobre a possibilidade de sustentarem água líquida em sua superfície, em paralelo à aprendizagem acerca de diagramas de fase. Nesta figura, as fases mais estáveis da água (sólido, líquido e vapor) são separadas por curvas que definem as regiões nas quais duas fases coexistem em equilíbrio termodinâmico. A transição entre as fases líquida e gasosa termina em um ponto especial conhecido como ponto crítico, que na água está localizado em $T_{c}=374,4^{\circ} \mathrm{C}$ e $P_{c}=22,242 M P a$. Os pontos no gráfico em que as condições de temperatura e pressão permitem a coexistência de três fases são chamados de ponto triplo. Os estados de gelo, líquido e vapor estão em equilíbrio no ponto triplo, localizado a $273,16 \mathrm{~K}$ e $611,0 \mathrm{~Pa}$, sendo o primeiro valor utilizado para definir a unidade de temperatura Kelvin [16].

Em seguida discutiremos as condições de temperatura e pressão em cada astro (planeta ou exoplaneta), sempre contextualizando-os em relação à figura 5 e, quando for o caso, comparando temperaturas reais médias com as temperaturas efetivas, ilustradas na figura 3 Note que as temperaturas mínimas e máximas observadas foram indicadas para alguns dos astros na figura 5

Na figura 3 percebe-se que o planeta Mercúrio possui uma temperatura observada menor do que aquela obtida pela equação 4 sendo que o valor observado é uma média das temperaturas nos lados do planeta considerados quente e frio. Isto ocorre porque este é o planeta do sistema solar que apresenta a maior variação de temperatura média na superfície, com valores que chegam a $427^{\circ} \mathrm{C}$ durante o dia e $-173^{\circ} \mathrm{C}$ durante a noite 17 . Esta intensa variação ocorre devido a uma relação exclusiva entre o período de rotação e o período orbital do planeta $(3: 2)$, onde a cada três rotações em torno do seu eixo, Mercúrio completa duas voltas ao redor do Sol. Desta forma, um dia solar (nascer ao pôr do Sol) tem duração de 1,5 anos em Mercúrio ou 132 dias terrestres. Considera-se que essa característica foi adquirida naturalmente como consequência do gradiente gravitacional do Sol, que diminuiu a velocidade de rotação do planeta, fazendo com que a relação entre os valores dos períodos de rotação e orbital seja de 1, 510 É o segundo planeta mais denso do Sistema Solar e, em vez de uma atmosfera, possui uma fina exosfera na qual átomos e moléculas raramente colidem. Possui um fraco campo magnético como consequência do movimento entre o núcleo líquido e o manto sólido 18,19. Imagens do sistema de radar Goldstone já revelaram a existência de regiões altamente refletoras no polo norte de Mercúrio, sugerindo a presença de depósitos de gelo no interior de profundas crateras polares do planeta, como resultado de um contínuo bombardeamento de micrometeoritos pelos últimos 3,5 bilhões de anos [20].

De acordo com a figura 3 Mercúrio deveria ser o planeta mais quente do Sistema Solar, seguido por Vênus. Entretanto, mesmo sendo considerado o planeta mais parecido com a Terra em relação à massa e volume, Vênus

${ }^{10}$ Mercúrio apresenta um período de rotação de 58,64 dias, enquanto seu período orbital é de 88 dias

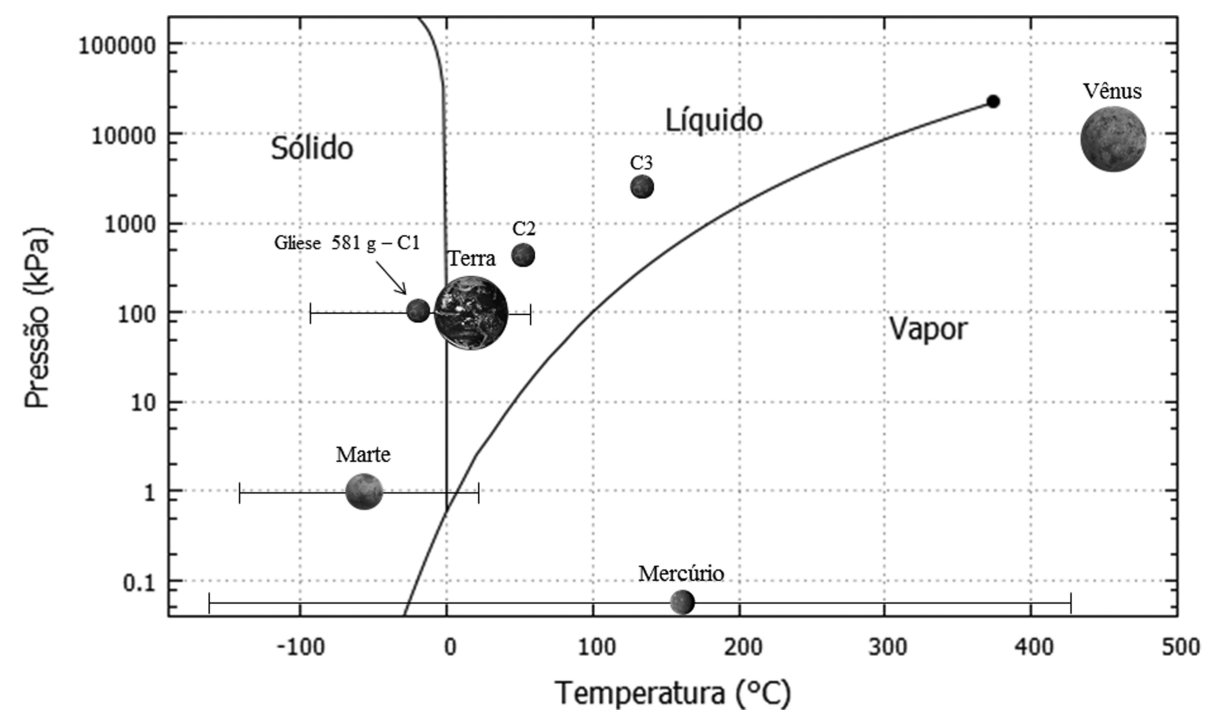

Figura 5: Diagrama de fases da água localizando os planetas rochosos do sistema solar e candidato a exoplanetas Gliese 581g. 
possui condições superficiais drasticamente diferentes das do nosso planeta. Possui uma temperatura observada aproximada de $462^{\circ} \mathrm{C}$ devido à composição de sua atmosfera, formada principalmente por $\mathrm{CO}_{2}(96,5 \%)$, $N_{2}(3,5 \%)$, nuvens de ácido sulfúrico e traços de vapor de água, sendo que este último é continuamente extraído da atmosfera pelo vento solar, devido à ausência de um campo magnético. A espessa camada atmosférica de Vênus aprisiona o calor proveniente do Sol causando um intenso efeito estufa no planeta, resultando em uma temperatura alta o suficiente para derreter chumbo 21. A elevada quantidade de dióxido de carbono também é responsável pela alta pressão atmosférica, que chega a 9,2 MPa. Apesar da lenta rotação (-5.832, 4 horas $\left.{ }^{11}\right)$ Vênus possui ventos que se deslocam a elevadas velocidades distribuindo o calor de forma uniforme, fazendo com que a temperatura seja basicamente a mesma em toda a superfície do planeta [20].

Um conjunto de fatores planetários e astronômicos ideais permitiram a existência de pressões e temperaturais compatíveis com a existência de água líquida na superfície da Terra pelos últimos 3,8 bilhões de anos. Além de tornar o nosso planeta único, a presença de vida na Terra tem exercido uma grande influencia na composição atmosférica da Terra, visto que se não fosse por ela, a nossa atmosfera poderia apresentar grandes semelhanças com a de Vênus e Marte. Há uma possibilidade de que no início da sua formação a Terra possuísse grandes quantidades de dióxido de carbono assim como Vênus, porém as atividades metabólicas dos seres vivos retiraram o carbono da atmosfera utilizando-o para a formação de seus tecidos. As atividades metabólicas dos seres vivos tem uma influência direta na atmosfera e no albedo do planeta, ocasionando uma temperatura observada inferior à temperatura efetiva. Juntamente com compostos de carbono, a água e o nitrogênio representam os principais constituintes da crosta terrestre. Cerca de três quartos da superfície são cobertos por oceanos que podem chegar a uma profundidade de 4 quilômetros, enquanto o nitrogênio $\left(N_{2}\right)$ representa $78 \%$ da atmosfera terrestre 20,21.

Apresentando temperaturas menores e apenas $1 \%$ da pressão atmosférica da Terra, Marte não possui condições de sustentar água líquida em sua superfície, permitindo apenas transições diretas do estado sólido para o vapor 22]. O planeta possui uma atmosfera composta por dióxido de carbono (95\%), nitrogênio (3\%) e vapor de água. Além disso, as calotas polares deste planeta são formadas por água e dióxido de carbono congelado, ambos altamente reflexivos, e o planeta não possui campo magnético, o que contribui para a retirada de gases voláteis compostos por hidrogênio de sua atmosfera [20]. A junção destes fatores colaborou para que a superfície do planeta esteja atualmente em uma temperatura me-

\footnotetext{
${ }^{11}$ Vênus possui um sentido de rotação contrário aos outros planetas do Sistema Solar, girando de leste para oeste
}

nor do que aquela permitida pela radiação através da temperatura efetiva.

Marte possui um período de rotação parecido com a Terra e também apresenta uma pequena inclinação em seu eixo de rotação em relação ao plano de translação, o que o faz apresentar estações diferenciadas em seus hemisférios norte e sul. Desde os primeiros registros fotográficos da superfície do planeta obtidos na década de sessenta, este planeta tem sido alvo de inúmeras missões espaciais que tem revelado que apesar do seu aspecto atual ser seco e sujeito à intensas radiações devido ao vento solar, o planeta pode ter possuído um clima menos hostil, possivelmente muito parecido com o clima terrestre. Informações recentes sobre a possível existência de água líquida no passado de Marte tem contribuído para a hipótese de que em tempos primitivos o planeta garantisse condições para a existência de vida [23].

$\mathrm{Na}$ data atual foram detectados cerca de 44 planetas que se encontram na zona habitável de sistemas estelares em nossa galáxia [24]. A reivindicação da primeira detecção de um exoplaneta na zona habitável ocorreu no ano de 2010 [25], quando uma análise de dados usando o método da velocidade radial indicou a existência do planeta Gliese 581g, orbitando a estrela do tipo M Gliese 581, na constelação de Libra. A detecção de Gliese 581g é controversa, sendo motivo de debate na literatura 25], mas simulações computacionais sinalizam que uma superTerra, ou seja, um planeta com algumas vezes a massa da Terra, pode ser estável em órbitas com diferentes níveis de excentricidade, além da órbita circular [26]. No entanto, por ser (potencialmente) o primeiro exoplaneta detectado na zona habitável, diversas simulações computacionais foram realizadas visando criar cenários para a atmosfera deste candidato a exoplaneta 14,27,28. Em um trabalho recente foram obtidos padrões de temperatura para 20 cenários distintos envolvendo variações na pressão superficial e na concentração de $\mathrm{CO}_{2}$, utilizando um modelo matemático para uma atmosfera de nitrogênio e dióxido de carbono, considerando trocas de calor e radiação térmica estelar, em uma aproximação que desconsidera a presença de nuvens [14]. Os resultados obtidos com este modelo mostram que, caso Gliese $581 \mathrm{~g}$ de fato exista, é possível que água líquida esteja presente na superfície do candidato a exoplaneta para uma grande variedade de cenários. Na figura 5 indicamos três cenários possíveis para os valores médios de temperatura e pressão em Gliese 581g, extraídos da ref. [14]. O primeiro cenário (C1), similar à Terra, contempla uma pressão superficial de uma atmosfera e $355 \mathrm{ppm}$ de $\mathrm{CO}_{2}$, resultando em uma temperatura média de $-19^{\circ} \mathrm{C}$. No segundo cenário (C2) a pressão superficial é de 5 atm com $5 \%$ de $\mathrm{CO}_{2}$ e a temperatura superficial média resultante é de $44^{\circ} \mathrm{C}$. Por fim, o último cenário (C3) que ilustramos contempla uma pressão igual a 20 atm na superfície com $95 \%$ de $\mathrm{CO}_{2}$ na atmosfera e uma temperatura média de $128^{\circ} \mathrm{C}$. Os cenários C2 e C3 indicam que as condições médias de temperatura e pressão de Gliese $581 \mathrm{~g}$ permitem a pre- 
sença de água na fase líquida, mas mesmo no cenário $\mathrm{C} 1$ podemos considerar que é possível a existência de água líquida se considerarmos que o modelo descreve condições médias para a temperatura e que mesmo pequenas variações na temperatura, como as encontradas na Terra, são suficientes para permitir a existência de água líquida no equador ou abaixo de camadas superficiais de gelo.

\section{Conclusões}

Diante dos desafios apresentados pelo ensino de termodinâmica nos ensinos fundamental e médio, neste estudo apresentamos uma proposta na qual a integração de diferentes áreas do conhecimento podem possibilitar uma abordagem mais complexa de temas que geralmente são trabalhados apenas de forma superficial. A utilização de conceitos de astrobiologia, uma área de estudo que desperta a curiosidade dos estudantes e que a cada dia vem conquistando mais espaço no meio científico e na mídia, é uma ferramenta que pode contribuir para um ensino mais contextualizado e integrado de temas da termodinâmica.

A discussão acerca das temperaturas reais e efetivas dos planetas rochosos do sistema, assim como a análise das condições médias de temperatura e pressão dos astros sobreposta ao diagrama de fases da água, permite que os estudantes desenvolvam um raciocínio crítico quanto à possibilidade dos planetas sustentarem água líquida em sua superfície, bem como suas possíveis condições para a habitabilidade.

Devido ao seu caráter interdisciplinar, a astrobiologia pode ser utilizada como potencializadora nos processos de ensino-aprendizagem, facilitando o entendimento de conteúdos de disciplinas como química, biologia e geologia, ilustrando o caráter interdisciplinar destas áreas do conhecimento e permitindo uma ampliação na compreensão das condições que possibilitam o surgimento e manutenção da vida.

\section{Agradecimentos}

Este trabalho foi originalmente inspirado por uma série de palestras sobre "A origem da vida", ministradas por um grupo interdisciplinar de professores da Faculdade UnB Planaltina. Agradecemos Paulo Eduardo de Brito e Dulce Maria da Rocha por numerosas discussões calorosas sobre a vida no universo e sua origem. Agradecemos ao Programa de Educação Tutorial do MEC e à Universidade de Brasília pelo apoio financeiro.

\section{Referências}

[1] F. Casoli e T. Encrenaz, The New World (Springer, Chichester, 2007) p. 3.

[2] L. Bernardes, Exoplanetas, Extremófilos e Habitabilidade. Dissertação de Mestrado, Universidade de São Paulo, 2013
[3] Disponível em "Nasa Exoplanet Archive", http: //exoplanetarchive.ipac.caltech.edu/, acesso em $10 / 11 / 2016$.

[4] R. Barnes, V.S. Meadows e N. Evans, The Astrophysical Journal 814, 91 (2015).

[5] Disponível em "Kepler: A Search for Habitable Planets", https://kepler.nasa.gov, acesso em 10/11/2016.

[6] S.C. Aroca e C.C. Silva, Revista Brasileira de Ensino de Física 33, e1402 (2011).

[7] E.R. Mozena e F. Ostermann, Revista Brasileira de Ensino de Física 36, e1403 (2014).

[8] G. Gonzalez e D. Brownlee, The Galactic Habitable Zone I . Galactic Chemical Evolution. Master's Thesis, University of Washington, 2001.

[9] C.S. Cockell, T. Bush, C. Bryce, S. Direito, M. FoxPowell, J. Harrison, H. Lammer, H. Landenmark, J. Martin-Torres, N. Nicholson, et al., Astrobiology 16, 89 (2016).

[10] J.A. Baross, The Limits of Organic Life in Planetary Systems. (The National Academies Press, Washington,D.C., 2007).

[11] J.F. Kasting, D. Whitmire e R.T. Reynolds, Icarus 101, 108 (1993).

[12] Y.S. Lee e M. Richmond, Journal of Emerging Investigators 2013, 1 (2013).

[13] F. Gewandsznajder, Ciências: O Planeta Terra (Editora Ática, São Paulo, 2011), $4^{\mathrm{a}}$ ed, p. 232.

[14] P. Paris, S. Gebauer, M. Godolt, H. Rauer e B. Stracke, Astronomy \& Astrophysics 532, A58 (2011).

[15] Disponível em "Our Solar System: Overview: Our Galactic Neighborhood": https://solarsystem.nasa.gov/ planets/solarsystem, acesso em 10/11/2016.

[16] P.W. Atkins, Princípios de Química: Questionando a Vida Moderna e o Meio Ambiente (Bookman, Porto Alegre, 2006), $3^{\mathrm{a}}$ ed.

[17] Disponível em "Solar System Exploration": http: //solarsystem.nasa.gov/planets/mercury/facts, acesso em 10/11/2016.

[18] S. J. Peale, The Rotational Dynamics of Mercury and the State of its Core, Technical Report (University of California, Santa Barbara, 1988).

[19] R.G. Strom, Science 19, 1471 (1997).

[20] A. Hanslmeier, Water in the Universe (Springer, New York, 2011).

[21] L. Margulis, Environmental Evolution: Effects of the Origin and Evolution of Life On Planet Earth (MIT Press, Cambridge, 2000), 3rd ed.

[22] B.M. Jakosky e R.J. Phillips, Nature 412, 237 (2001).

[23] F. Gómez, J.A. Rodríguez-Manfredi, N. Rodríguez, M. Fernández-Sam Pedro, F.J. Caballero-Castrejón and R. Amils, Planetary and Space Science 68, 48 (2012).

[24] Disponível em "Habitable Exoplanets Catalog": http://phl.upr.edu/projects/habitableexoplanets-catalog, acesso em 10/11/2016.

[25] Y. Bhattacharjee, Science 337, 398 (2012).

[26] Z. Tóth e I. Nagy, Oxford Journals 442, 454 (2014).

[27] R.D. Wordsworth, F. Forget, F. Selsis, J.-B. Madeleine, E. Millour e V. Eymet, Astronomy \& Astrophysics 522, A22 (2010).

[28] R.T. Pierrehumbert, The Astrophysical Journal 726, L8 (2011). 